\title{
Safety and efficacy of deep brain stimulation in refractory cluster headache: a randomized placebo-controlled double-blind trial followed by a 1-year open extension
}

\author{
Denys Fontaine $\cdot$ Yves Lazorthes Patrick Mertens $\cdot$ Serge Blond $\cdot$ \\ Gilles Géraud · Nelly Fabre • Malou Navez • Christian Lucas • \\ Francois Dubois $\cdot$ Sebastien Gonfrier $\cdot$ Philippe Paquis $\cdot$ Michel Lantéri-Minet
}

Received: 21 September 2009/Accepted: 27 October 2009/Published online: 21 November 2009

(c) Springer-Verlag 2009

\begin{abstract}
Chronic cluster headache (CCH) is a disabling primary headache, considering the severity and frequency of pain attacks. Deep brain stimulation (DBS) has been used to treat severe refractory $\mathrm{CCH}$, but assessment of its efficacy has been limited to open studies. We performed a prospective crossover, double-blind, multicenter study assessing the efficacy and safety of unilateral hypothalamic DBS in 11 patients with severe refractory $\mathrm{CCH}$. The randomized phase compared active and sham stimulation during 1-month periods, and was followed by a 1-year open phase. The severity of $\mathrm{CCH}$ was assessed by the weekly attacks frequency (primary outcome), pain intensity, sumatriptan injections, emotional impact (HAD) and
\end{abstract}

D. Fontaine $\cdot$ P. Paquis

Department of Neurosurgery,

Centre Hospitalier Universitaire de Nice,

Nice, France

e-mail: fontaine.d@chu-nice.fr

Y. Lazorthes

Department of Neurosurgery,

Centre Hospitalier Universitaire de Toulouse,

Toulouse, France

P. Mertens

Department of Neurosurgery,

Centre Hospitalier Universitaire de Lyon,

Lyon, France

S. Blond

Department of Neurosurgery,

Centre Hospitalier Universitaire de Lille,

Lille, France

G. Géraud · N. Fabre

Department of Neurology,

Centre Hospitalier Universitaire de Toulouse,

Toulouse, France quality of life (SF12). Tolerance was assessed by active surveillance of behavior, homeostatic and hormonal functions. During the randomized phase, no significant change in primary and secondary outcome measures was observed between active and sham stimulation. At the end of the open phase, 6/11 responded to the chronic stimulation (weekly frequency of attacks decrease $>50 \%$ ), including three pain-free patients. There were three serious adverse events, including subcutaneous infection, transient loss of consciousness and micturition syncopes. No significant change in hormonal functions or electrolytic balance was observed. Randomized phase findings of this study did not support the efficacy of DBS in refractory $\mathrm{CCH}$, but open
M. Navez

Pain Department, Centre Hospitalier Universitaire de Saint-Etienne, Saint Etienne, France

C. Lucas

Department of Neurology, Centre Hospitalier Universitaire de Lille, Lille, France

F. Dubois

Pain Department,

Centre Hospitalier Universitaire de Lille, Lille, France

S. Gonfrier

Department of Statistics, Centre Hospitalier Universitaire de Nice, Nice, France

M. Lantéri-Minet

Pain Department, Centre Hospitalier Universitaire de Nice, Nice, France

M. Lantéri-Minet ( $\square)$

Département d'Evaluation et Traitement de la Douleur,

Hopital Pasteur, 30 avenue de la Voie Romaine,

06000 Nice, France

e-mail: lanteri-minet.m@chu-nice.fr 
phase findings suggested long-term efficacy in more than $50 \%$ patients, confirming previous data, without high morbidity. Discrepancy between these findings justifies additional controlled studies (clinicaltrials.gov number NCT00662935).

Keywords Cluster headache - Deep brain stimulation . Hypothalamus · Headache · Pain modulation .

Randomized trial

\section{Introduction}

Cluster headache $(\mathrm{CH})$ is a primary headache and belongs to the group of the trigeminal autonomic cephalalgias in the International Classification of the Headaches Disorders [1-3]. CH mainly affects men and is characterized by strictly unilateral short-lasting pain attacks associated with prominent parasympathetic features. Episodic $\mathrm{CH}$ affects $80-90 \%$ of patients who describe periods of attacks (cluster) and periods of remission. Chronic $\mathrm{CH}$ (unremitting from onset or evolved from episodic form) lacks the remissions and is diagnosed after 1 year without remission or with remission periods lasting less than 1 month [2]. $\mathrm{CH}$ is one of the most painful conditions in humans and is often referred to as "suicidal headache". The pathophysiology of $\mathrm{CH}$ revolves around the trigeminal-autonomic reflex whose activation explains the trigeminal topography of pain and the ipsilateral autonomic features [4]. Functional imaging demonstrated a brain activation during attacks [5], co-localized with a structural change, in the posterior hypothalamic region. [6]. This prompted the use of deep-brain stimulation (DBS) to modulate this region in a patient with refractory chronic $\mathrm{CH}$ which led to complete relief from attacks [7]. Based on this observation, DBS was introduced in the treatment of medically refractory chronic $\mathrm{CH}$. After 8 years of experience, DBS is claimed to be successful in controlling the pain attacks in about $60 \%$ of the 41 chronic $\mathrm{CH}$ patients implanted worldwide [8-14]. Yet such a claim may be debatable considering the absence of formal blinded controlled study. Here, we report the first randomized, double-blind, crossover study comparing DBS (stimulation "On") of the posterior hypothalamic region with a sham control (stimulation "Off"), followed by a 10-month open phase (stimulation "On") with a special focus on the procedure's safety.

\section{Methods}

\section{Patients}

Patients with refractory chronic $\mathrm{CH}$ were enrolled in the study according to the following inclusion criteria: chronic
$\mathrm{CH}$ according to ICHD-II criteria [2]; disease duration over 3 years; resistance to pharmacological prophylactic treatment with adequate trials (verapamil up to $960 \mathrm{mg} /$ day, lithium with plasma level from 0.6 to $1 \mathrm{mEq} / \mathrm{l}$, association of both; in absence of adverse events); daily attacks; absence of substance abuse or dependence; age 18-65year-old; normal findings on magnetic resonance imaging; no contraindications to surgery or anesthesia.

\section{Study design}

This study consisted of a randomized, double-blind, crossover design with two 1-month periods separated by a 1-week wash-out period and an extension 10-month open phase (Fig. 1). The trial was conducted in four academic centers in accordance with the Declaration of Helsinki, and was approved by the ethics committee of the Nice University Hospital (Comité de Protection des Personnes Sud Méditerranée V). All centers associated a neurological team belonging to the "Observatoire des Migraines et Céphalées" set up by the French Headache Society [15] and a neurosurgical team highly qualified in the DBS domain and pain management.

All patients provided written informed consent. Eligible patients were randomly assigned in a 1:1 ratio to one of the two groups; either active stimulation followed by a shamstimulation period (On-Off) or the reverse other (Off-On). Previous studies $[9,11,12]$ demonstrated that posterior hypothalamic stimulation does not induce perceptible sensations, allowing double-blind trial as the patient is not able to identify the "on" or "off" condition. We used a blocking scheme randomization and a central randomization procedure without stratification. Stimulation parameters were set up by the neurosurgeon. The 1-month duration of the randomized periods was defined according to the data available when the study was designed. At that time, in 2003, in the study of Franzini et al. [9], "pain disappearance ... occurred after few hours in 2 cases and later (1-4 weeks) in the other 3 cases". In the study of Schoenen et al. [11], "all patients improved 2 weeks after implantation". Clinical evaluation was performed by the neurologist blinded of the stimulation status. At each evaluation, clinical data collected were: number of attacks during the last week (calculated from the individual patient's diary), mean attack intensity during the last week (according to Likert scale), number of subcutaneous sumatriptan administration during the last week (from the patient's diary), oxygen use (yes or no), anxiety and depression levels (Hospital Anxiety Depression scale), quality of life (SF-12 scale), supine and standing blood pressure, heart rate, weight and body temperature. Electrolyte balance and hormonal functions (thyroid hormones, TSH, ACTH, cortisol, SDHEA, insulin, prolactin, testosterone, estradiol, 


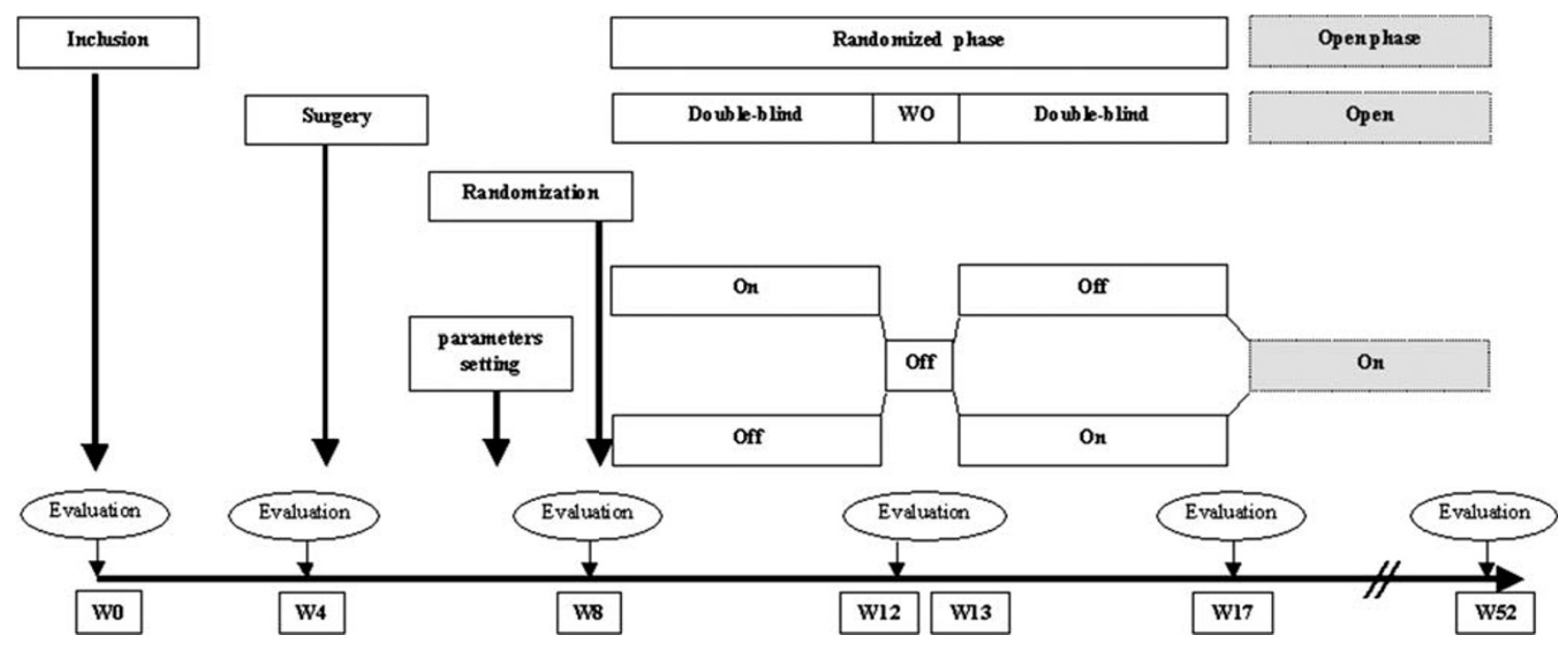

Fig. 1 Design of the study. The randomized phase of the study included two 1-month treatment periods (week 8 to week 12 and week 13 to week 17) separated by a 1-week washout period. Patients were evaluated at inclusion, 1 week before surgery; 4 weeks after surgery

LH, FSH, GH and IGF-1) were evaluated at each evaluation. After surgery, evaluations additionally included: patient's satisfaction (Patient's Global Impression of Change) and changes in thirst, appetite, libido, sleepwalking cycle and behavior. Any new symptom or worsening of a preexisting symptom was classified as an adverse event. An adverse event was classified as serious in case of death, hospitalization, sequel or consideration as serious by the clinician. According to the study protocol, prophylactic treatment was held constant during the randomized phase, but could be adapted during the open phase.

\section{Surgery and stimulation}

The posterior hypothalamus was targeted on preoperative 3D MRI, according to previously published coordinates, namely $2 \mathrm{~mm}$ lateral to the midline, $3 \mathrm{~mm}$ posterior and $5 \mathrm{~mm}$ below the mid-commissural point [9]. The fourcontact electrode (model 3389 DBS, Medtronic) was implanted stereotactically (deepest contact on the target), ipsilateral to the pain side, under local anesthesia, without intraoperative micro-recording (Fig. 2). Intraoperative test stimulation (up to $3 \mathrm{~V}$ ) was conducted through this electrode before its fixation to check any side effect. The electrode location was confirmed by postoperative 3D neuroimaging before its connection to the pulse generator (Kinetra, Medtronic), implanted under general anesthesia. Optimal stimulation parameters were defined by the neurosurgeon during the week following surgery before the randomization. The closest contact from the theoretical target on postoperative imaging was used for stimulation in (before active or sham stimulation) and at the end of the first randomization period (weeks 12 and 17). This randomized phase was followed by a 10-month open phase. Patients were evaluated at the end of this phase (week 52)

the randomized phase. Stimulation frequency and pulse duration were, respectively, $185 \mathrm{~Hz}$ and $60 \mu \mathrm{s}$. Voltage was individually adjusted according to side effects investigated by increasing voltage: $3 \mathrm{~V}$ by default or $80 \%$ of the threshold producing side effects. These stimulation parameters were kept constant during all along the randomized phase, but could be changed during the open phase.

\section{Outcome and statistical analysis}

All outcome measures were analyzed by intention to treat. We performed a crossover analysis for the On and Off periods. Primary outcome was the number of attacks during the last week of each period, according to the International Headache Society guidelines for controlled trials of drugs in $\mathrm{CH}$ [16]. Secondary outcomes were the number of subcutaneous sumatriptan administration during the last week, intensity of attacks, satisfaction of patients, HAD sub-scores and SF-12 scores. Review of the early DBS studies $[9,11]$ in $\mathrm{CH}$, available when the present study has been designed, did not allow to find the mean and variability of our primary outcome, namely frequency of attacks per week, in this refractory $\mathrm{CH}$ patients candidates for surgery. Due to absence of published data, this estimate was based on the characteristics of refractory chronic $\mathrm{CH}$ patients registered in the Nice University Hospital database. Power calculation was based on our estimate that at baseline mean weekly frequency of attacks would be 23.9 (SD 3.7). The study was designed to have an overall power of $90 \%$ to detect a $50 \%$ reduction of the primary endpoint during the stimulation period. According to Jones and Kenward [17], three effects were tested: carry-over, period 

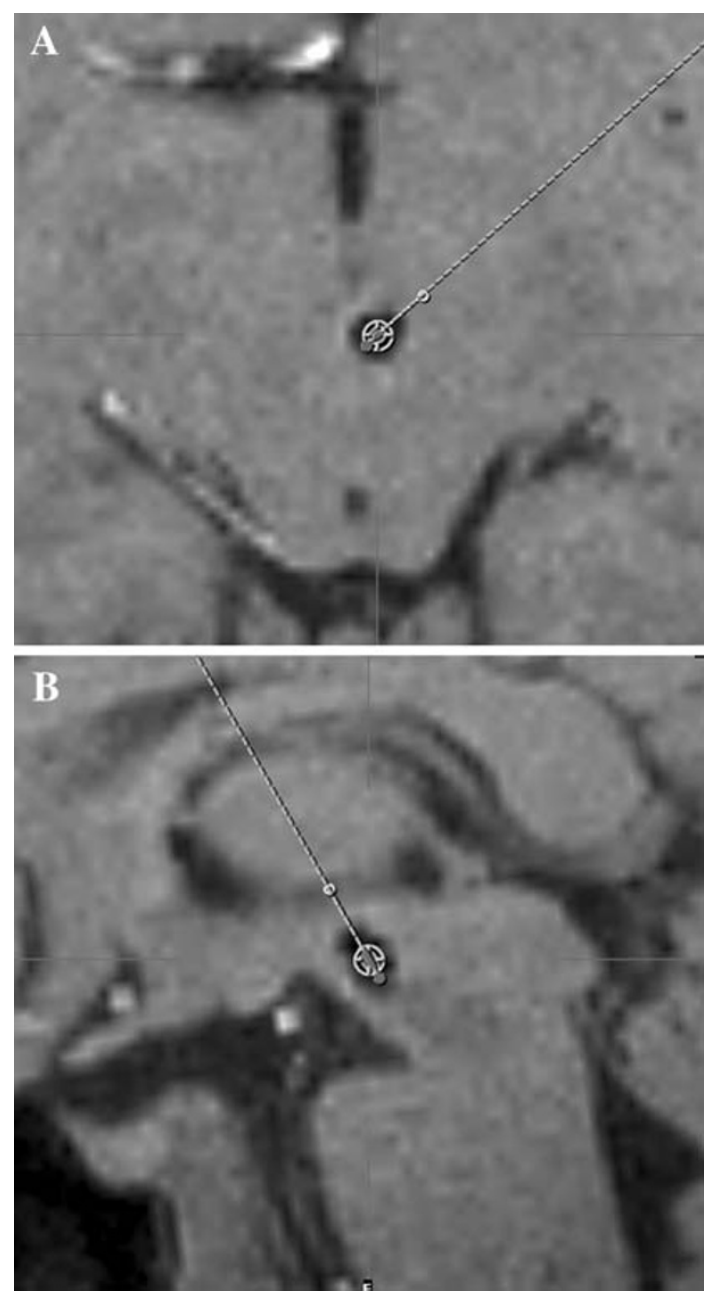

Fig. 2 Postoperative tridimensional MRI (patient C1P4), T1 weighted images after gadolinium injection, axial (a) and saggital (b) slices, showing the location of the stimulating contact (white circle) within the black artifact generated by the electrode. Dotted line indicates the projection of the electrode trajectory on the slice

and treatment effects. Type I error was fixed at $10 \%$ for the carry-over effect and 5\% for the others. Non-parametric two-sided Wilcoxon rank-sum tests were used for the analysis, given the number of patients. The effect of treatment at 1 year compared to baseline was done with a Wilcoxon test for paired samples (two-sided, type I error rate $=5 \%$ ) on primary and second outcomes. All the statistical analysis was performed using the SPSS version 11.0 program (SPSS Inc., Chicago, IL, USA).

\section{Results}

\section{Study population}

Twelve patients were included (May 2005-June 2007), 1 declined to participate, 11 were operated, 1 was explanted due to infection but re-implanted later, before the randomization. Consequently 11 patients completed the randomized phase and the open phase. Pre-implantation clinical characteristics of these 11 patients are shown in Table 1. According to the inclusion criteria, the patients included were non-responders to verapamil and lithium. However, considering the duration of chronic $\mathrm{CH}$, all these patients have been previously treated in sufficient dosages (unless contraindicated or unacceptable side effects) alone or in combination by gabapentine, indometacine, methysergide, pizotifen, topiramate, steroids and valproate, and were considered as non-responders. There was no significant difference in these characteristics between the two groups (On-Off and Off-On).

\section{Effect of electrode implantation}

Mean stereotactic coordinates (SD) of the deeper contact of the electrode relative to the mid-commissural point were $x=2.20$ (0.83), $y=-3.24$ (1.83) and $z=-3.69$ (1.71). There was no significant change in the mean weekly frequency of attacks after implantation compared to baseline, although two patients (C1P4 and C4P1) still showed a "lesion effect" (decrease $\geq 50 \%$ ) 1 month after surgery.

\section{Effect of the stimulation during the randomized phase}

At the end of the randomized phase, patients and neurologists were not able to identify their period allocation, confirming the double-blind evaluation. The weekly frequency of $\mathrm{CH}$ attacks did not significantly differ between the On and Off periods (Table 2). We did not detect any significant carry-over effect $(P=0.855)$ indicating that the effects of the first treatment period did not persist after the wash out. None of the secondary outcomes differed between stimulation and sham treatment. Stimulation voltages used during the randomized phase ranged from 1.0 to 2.8 .

Effect of the stimulation during the open phase

At the end of the 10-month open phase, the mean weekly attacks frequency decreased by $48.4 \%(P=0.08)$ and emotional impact was significantly reduced (Table 3 ). Other secondary outcomes did not change significantly. Six out of 11 patients were considered as responders (at least $50 \%$ decrease in weekly attacks frequency), including three pain-free patients (Fig. 3). Among these 6 patients, prophylactic treatment was stopped or dose-decreased $\geq 50 \%$ in 2, unchanged in 2 and modified in 2 (Table 4). We did not identify any predictive factor of efficacy in this small population, concerning clinical characteristics, stimulation parameters or electrode location. 
Table 1 Characteristics of the 11 patients before implantation

\begin{tabular}{|c|c|c|c|c|c|c|c|c|c|c|}
\hline $\begin{array}{l}\text { Center/ } \\
\text { patient no. }\end{array}$ & Group & Sex & $\begin{array}{l}\text { Age } \\
\text { (years) }\end{array}$ & $\begin{array}{l}\text { Disease } \\
\text { duration } \\
\text { (years) }\end{array}$ & Attack side & $\begin{array}{l}\text { Onset } \\
\text { clinical } \\
\text { form }\end{array}$ & $\begin{array}{l}\text { Attacks/ } \\
\text { week }\end{array}$ & $\begin{array}{l}\text { Pain } \\
\text { intensity }\end{array}$ & $\begin{array}{l}\text { Sumatriptan } \\
\text { injection/ } \\
\text { week }\end{array}$ & $\begin{array}{l}\text { Oxygen } \\
\text { use }\end{array}$ \\
\hline $\mathrm{C} 1 / \mathrm{P} 1$ & On/Off & M & 52 & 35 & Left & Episodic & 14 & 9 & 1 & No \\
\hline $\mathrm{C} 1 / \mathrm{P} 2$ & Off/On & M & 40 & 12 & Right & Chronic & 14 & 5 & 14 & No \\
\hline $\mathrm{C} 1 / \mathrm{P} 3$ & Off/On & M & 51 & 8 & Left & Episodic & 19 & 2 & 15 & No \\
\hline $\mathrm{C} 1 / \mathrm{P} 4$ & On/Off & M & 44 & 10 & Left & Chronic & 28 & 10 & 0 & No \\
\hline $\mathrm{C} 1 / \mathrm{P} 5$ & On/Off & M & 47 & 7 & Right & Chronic & 11 & 6 & 11 & No \\
\hline $\mathrm{C} 2 / \mathrm{P} 1$ & Off/On & M & 50 & 20 & Right & Episodic & 20 & 5 & 0 & No \\
\hline $\mathrm{C} 2 / \mathrm{P} 2$ & Off/On & $\mathrm{F}$ & 42 & 3 & Left & Chronic & 7 & 8 & 1 & Yes \\
\hline $\mathrm{C} 3 / \mathrm{P} 1$ & On/Off & $\mathrm{F}$ & 42 & 7 & Right & Episodic & 53 & 6.5 & 0 & Yes \\
\hline $\mathrm{C} 3 / \mathrm{P} 2$ & Off/On & M & 36 & 7 & Left & Chronic & 9 & 5 & 11 & No \\
\hline $\mathrm{C} 4 / \mathrm{P} 1$ & Off/On & M & 39 & 18 & Right & Episodic & 14 & 5 & 14 & No \\
\hline $\mathrm{C} 4 / \mathrm{P} 2$ & On/Off & $\mathrm{F}$ & 43 & 6 & Right & Chronic & 7 & 7 & 1 & Yes \\
\hline Mean & & & 44.1 & 12.1 & & & 17.8 & 6.1 & 6.2 & \\
\hline
\end{tabular}

Some patients did not use attack treatment by sumatriptan and/or oxygen due to their lack of efficacy or side effects (such patients used opioids with weak efficacy)

\section{Adverse events}

Three serious adverse events were reported during the study, in two patients. One subcutaneous infection, 3 weeks after surgery, completely resolved after hardware removal and antibiotic treatment. The patient was re-implanted 6 months later. One patient experienced a preoperative loss of consciousness with hemiparesia shortly after test stimulation. An immediate CT-scan was normal. Symptoms spontaneously resolved in $2 \mathrm{~h}$ without sequel. During the open phase, the same patient reported multiple severe micturition syncopes associated with decrease of blood pressure in standing position.

Twenty-six non-serious adverse events (NSAE) were reported (Table 5). All of them were mild, and most of them were transient. Rates of NSAE were similar in both "On" and "Off" randomized periods. Compared to baseline, no change in electrolyte balance and hormonal levels were detected, except a testosterone level increase, observed in one patient during the "off" period and open phase. According to behavioral systematic auto-evaluation, $7 / 11$ patients reported a "calming effect" at the end of the open phase, compared to baseline.

\section{Discussion}

In the controlled phase of this study, we failed to demonstrate that DBS improved chronic $\mathrm{CH}$ when compared with sham stimulation. These findings contrasted with the results observed in the open phase of the study, which showed that more than $50 \%$ of the patients were improved over $50 \%$, and that mean attack frequency and emotional impact were markedly decreased. Our longterm results were similar with the overall outcome of the 38 patients with chronic $\mathrm{CH}$ previously implanted in noncontrolled conditions, showing that about $60 \%$ of them were improved over $50 \%$ [10, 14]. This improvement observed in the open phase was unlikely due to natural variations of the severity of chronic $\mathrm{CH}$, because the attack frequency had been stable for more than 3 years before the inclusion in the study. Open phase improvement was unlikely due to prophylactic treatment changes because in four out of six responders, this treatment was stopped, dose-decreased or unchanged. However, the improvement observed at the end of the open phase could be related to a sustained placebo effect, sometimes described in headache trials [18]. Although one could speculate that DBS is ineffective in chronic $\mathrm{CH}$, several bias, mainly related to the study design, might explain that efficacy of DBS has not been demonstrated in the randomized phase. First, the small sample size could have lead to inconclusive results in the randomized phase. Due to the lack of published data concerning this sub-population of refractory chronic $\mathrm{CH}$ patients, sample size calculation was based on the estimation of characteristics of these $\mathrm{CH}$ patients, registered in our institution database. Considering that the variability of weekly attack frequency was higher in the included population (SD: 13.2) than the estimated one (SD: 3.7), the sample size calculation might be a posteriori not adequate. Second, early publications, available when the study protocol has been written, mentioned that the delay between the stimulation onset and the therapeutic effect was less than 4 weeks, allowing to design a trial with 1 -month periods $[9,11]$. In later publications, this delay was longer (mean 


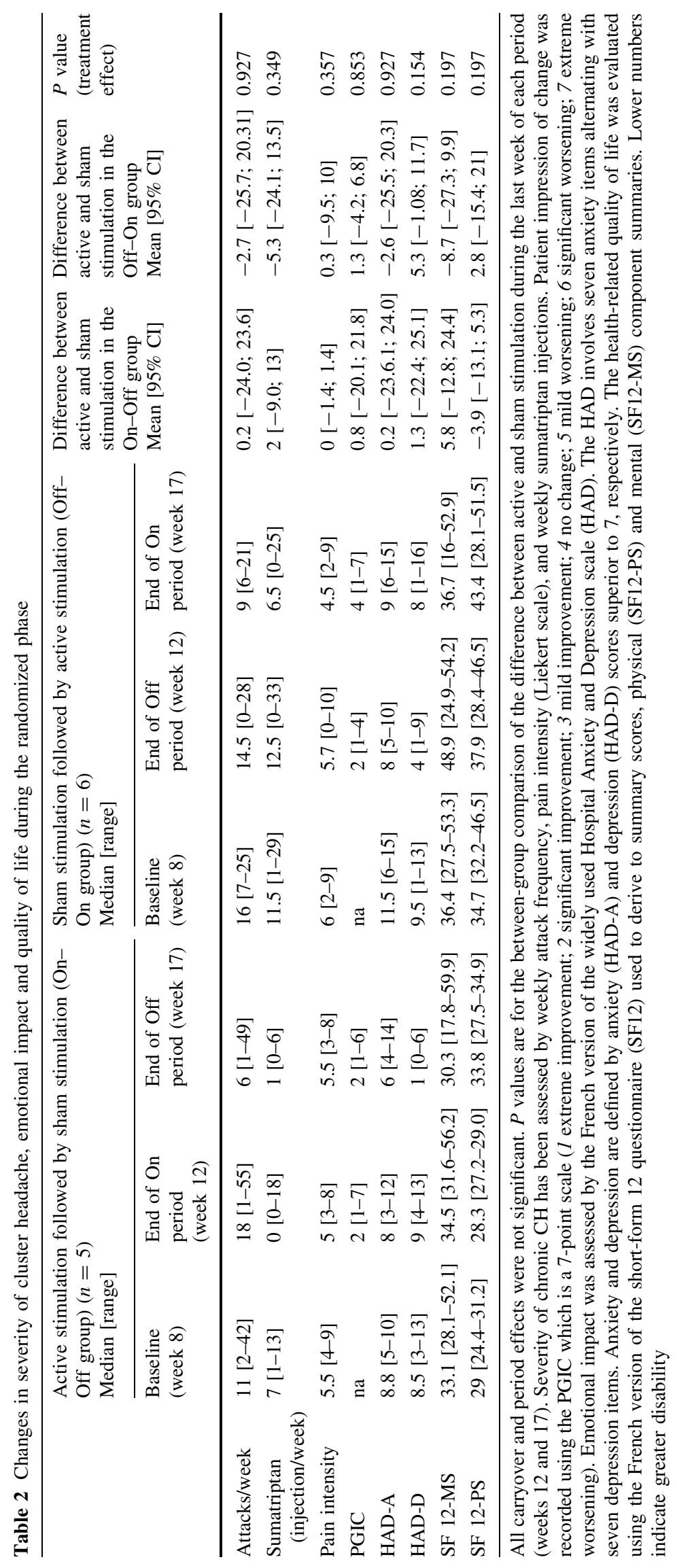


Table 3 Changes in severity of cluster headache, emotional impact and quality of life, between baseline (before surgery) and the end of the open phase

\begin{tabular}{lccc}
\hline & $\begin{array}{l}\text { Before implantation } \\
\text { (week 4) } \\
\text { Median [range] }\end{array}$ & $\begin{array}{l}\text { End of open phase } \\
\text { (week 52) } \\
\text { Median [range] }\end{array}$ & $\begin{array}{l}\text { Difference between end of } \\
\text { open phase and baseline } \\
\text { Mean [95\% CI] }\end{array}$ \\
\hline Attacks/week & $14[7 ; 53]$ & $8[0 ; 23]$ & $8.16[-18.3 ; 34.7]$ \\
Pain intensity & $6[2 ; 10]$ & $4.5[0 ; 10]$ & $1.1[-7.1 ; 9.3]$ \\
Sumatriptan & $1[0 ; 15]$ & $0.5[0 ; 26]$ & $-0.1[-11.3 ; 11.1]$ \\
$\quad$ injections/week) & $13[5 ; 18]$ & $7.5[0 ; 14]$ & $6.3[-5.1 ; 17.7]$ \\
HAD-A & $10[1 ; 16]$ & $4.5[1 ; 15]$ & $4.1[-6.48 ; 14.7]$ \\
HAD-D & $33.2[27.5 ; 53.3]$ & $37.0[20.7 ; 56.6]$ & $-0.6[-26.5 ; 25.2]$ \\
SF12-MS & $32.7[24.4 ; 46.5]$ & $39.7[25.2 ; 50.5]$ & $4.3[-16.7 ; 25.3]$ \\
SF12-PS & & 0.499 & 0.288 \\
\hline
\end{tabular}

Severity of chronic CH has been assessed by weekly attack frequency, pain intensity (Liekert scale), and weekly sumatriptan injections, during the last week before surgery and at the end of the open phase. Emotional impact was assessed by the French version of the widely used Hospital Anxiety and Depression scale (HAD). The HAD involves seven anxiety items alternating with seven depression items. Anxiety and depression are defined by anxiety (HAD-A) and depression (HAD-D) scores superior to 7, respectively. The health-related quality of life was evaluated using the French version of the short-form 12 questionnaire (SF12) used to derive to summary scores, physical (SF12-PS) and mental (SF12-MS) component summaries. Lower numbers indicate greater disability

Fig. 3 Individual changes in weekly attack frequency in the 11 patients between baseline (before surgery) and the end of the open phase

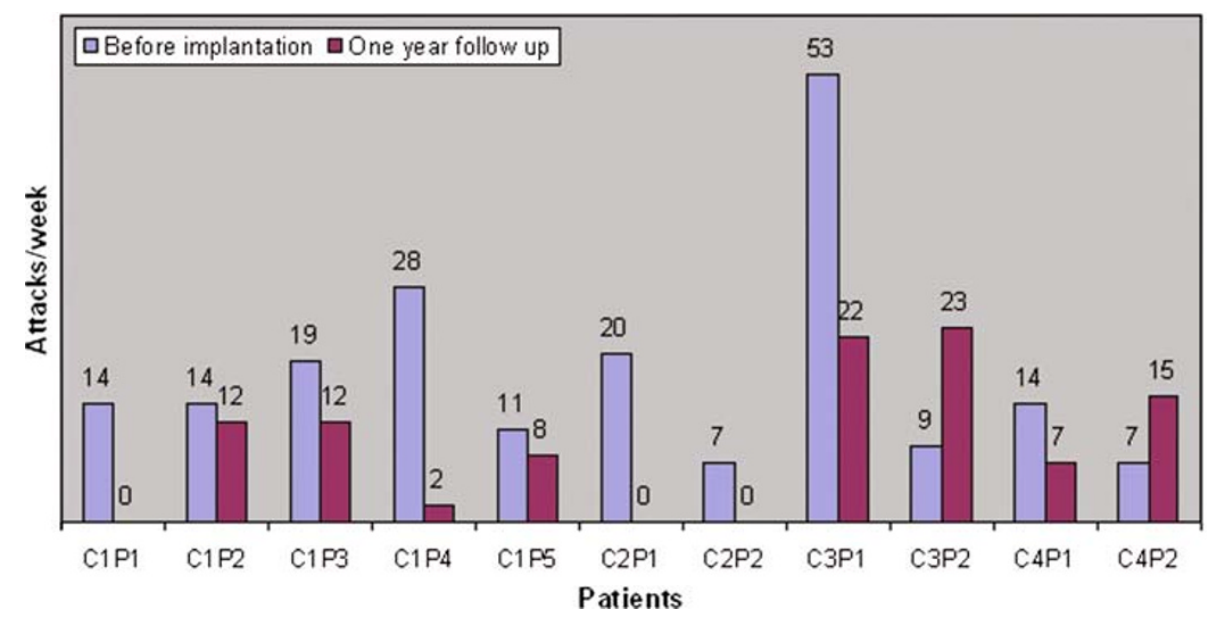

42 days) [19]. Consequently, a 1-month period might be too short to observe a significant improvement. Finally, stimulation parameters used during the randomized phase were set by default, based on the ones previously reported [9, 11]. During the open phase, tedious individual "trial and error"-based adjustment of these parameters allowed to reach the expected efficacy. Consequently, the randomized phase might have been conducted using nonoptimal parameters in some patients. Considering these possible biases, we proposed (after approval of the ethical committee) to the six responders in the open phase to be included in a new randomized phase, consisting in switching off the stimulator in control and double-blind conditions. All of them refused, fearing to loose the therapeutic effect.
DBS for $\mathrm{CH}$ appeared to be relatively safe in this study. No intracranial hemorrhage occurred, although this complication has been reported in early studies [11, 19]. In contrast to these studies, we did not use microelectrode recording in order to decrease the risk of bleeding, probably higher in this region. The most frequent adverse events (mainly visual disturbances) were stimulation-related and disappeared with stimulation parameters adjustment. Transient hemiparesia and loss of consciousness occurred during test stimulation in one patient, as in one case described by Starr et al. [12]. Micturition syncopes observed in one patient were probably related to changes in autonomic responses on cardiovascular system induced by chronic DBS [20]. No other clinically significant changes in homeostatic and hormonal functions were observed 
Table 4 Changes in prophylactic treatment (drug and daily dose) between baseline and the end of the open phase

\begin{tabular}{|c|c|c|c|}
\hline Patient & $\begin{array}{l}\text { Long-term } \\
\text { responder }\end{array}$ & $\begin{array}{l}\text { Before } \\
\text { implantation }\end{array}$ & 1-year follow-up \\
\hline C1P1 & Yes & $\begin{array}{l}\text { Verapamil } 240 \mathrm{mg} \\
\text { Lithium } 800 \mathrm{mg}\end{array}$ & No treatment \\
\hline $\mathrm{C} 1 \mathrm{P} 2$ & No & Verapamil $1440 \mathrm{mg}$ & Verapamil $1440 \mathrm{mg}$ \\
\hline C1P3 & No & Verapamil $1200 \mathrm{mg}$ & Verapamil $1200 \mathrm{mg}$ \\
\hline $\mathrm{C} 1 \mathrm{P} 4$ & Yes & $\begin{array}{l}\text { Verapamil } 600 \mathrm{mg} \\
\text { Lithium } 400 \mathrm{mg}\end{array}$ & Verapamil $1080 \mathrm{mg}$ \\
\hline C1P5 & No & $\begin{array}{l}\text { Verapamil } 720 \mathrm{mg} \\
\text { Lithium } 800 \mathrm{mg}\end{array}$ & No treatment \\
\hline $\mathrm{C} 2 \mathrm{P} 1$ & Yes & $\begin{array}{l}\text { Verapamil } 960 \mathrm{mg} \\
\text { Lithium } 1000 \mathrm{mg}\end{array}$ & $\begin{array}{l}\text { Verapamil } 360 \mathrm{mg} \\
\text { Lithium } 500 \mathrm{mg}\end{array}$ \\
\hline $\mathrm{C} 2 \mathrm{P} 2$ & Yes & Lithium $800 \mathrm{mg}$ & $\begin{array}{l}\text { Divalproex } 1500 \mathrm{mg} \\
\text { Fluoxetine } 40 \mathrm{mg}\end{array}$ \\
\hline $\mathrm{C} 3 \mathrm{P} 1$ & Yes & $\begin{array}{l}\text { Verapamil } 360 \mathrm{mg} \\
\text { Prednisone } 20 \mathrm{mg}\end{array}$ & Verapamil $360 \mathrm{mg}$ \\
\hline $\mathrm{C} 3 \mathrm{P} 2$ & No & Verapamil $480 \mathrm{mg}$ & Verapamil $480 \mathrm{mg}$ \\
\hline C4P1 & Yes & Verapamil $720 \mathrm{mg}$ & Verapamil $720 \mathrm{mg}$ \\
\hline $\mathrm{C} 4 \mathrm{P} 2$ & No & No & Verapamil $240 \mathrm{mg}$ \\
\hline
\end{tabular}

Long-term responders were defined as patients with weekly attack frequency decrease $\geq 50 \%$ at the end of the open phase, compared to baseline

despite an active surveillance of harms based on structured questionnaires and diagnostic tests performed at pre-specified time intervals all along the study.

$\mathrm{CH}$ has been described as the most painful primary headache, with a risk of suicide during attacks, justifying the moniker of "suicide headache" [21]. Considering our inclusion criteria and the patients' history (see "Study population"), all the patients included in our study fulfilled the criteria of intractable $\mathrm{CH}$ defined by international experts panels [22, 23], except for melatonin use (not available in France). In such patients, due to pain severity, absence of remission and treatment resistance, surgery may be a feasible option for pain control. Consequently, considering the safety and long-term outcomes of DBS in our study, the balance of benefit and harms may be considered as positive. This justifies to further evaluate DBS in additional controlled studies, using longer randomized periods or an initial open phase allowing enriched enrollment followed by a randomized phase. Predictors of outcome (headache characteristics, responses to medication and functional imaging features) need to be identified in order to select the potential responders [24]. However, future DBS studies should take into consideration the recent development of less invasive procedures, as occipital nerve subcutaneous stimulation (ONS) $[25,26]$. Although the ONS efficacy remains to be confirmed in controlled conditions, DBS may be reserved for failure of ONS.
Table 5 Adverse events

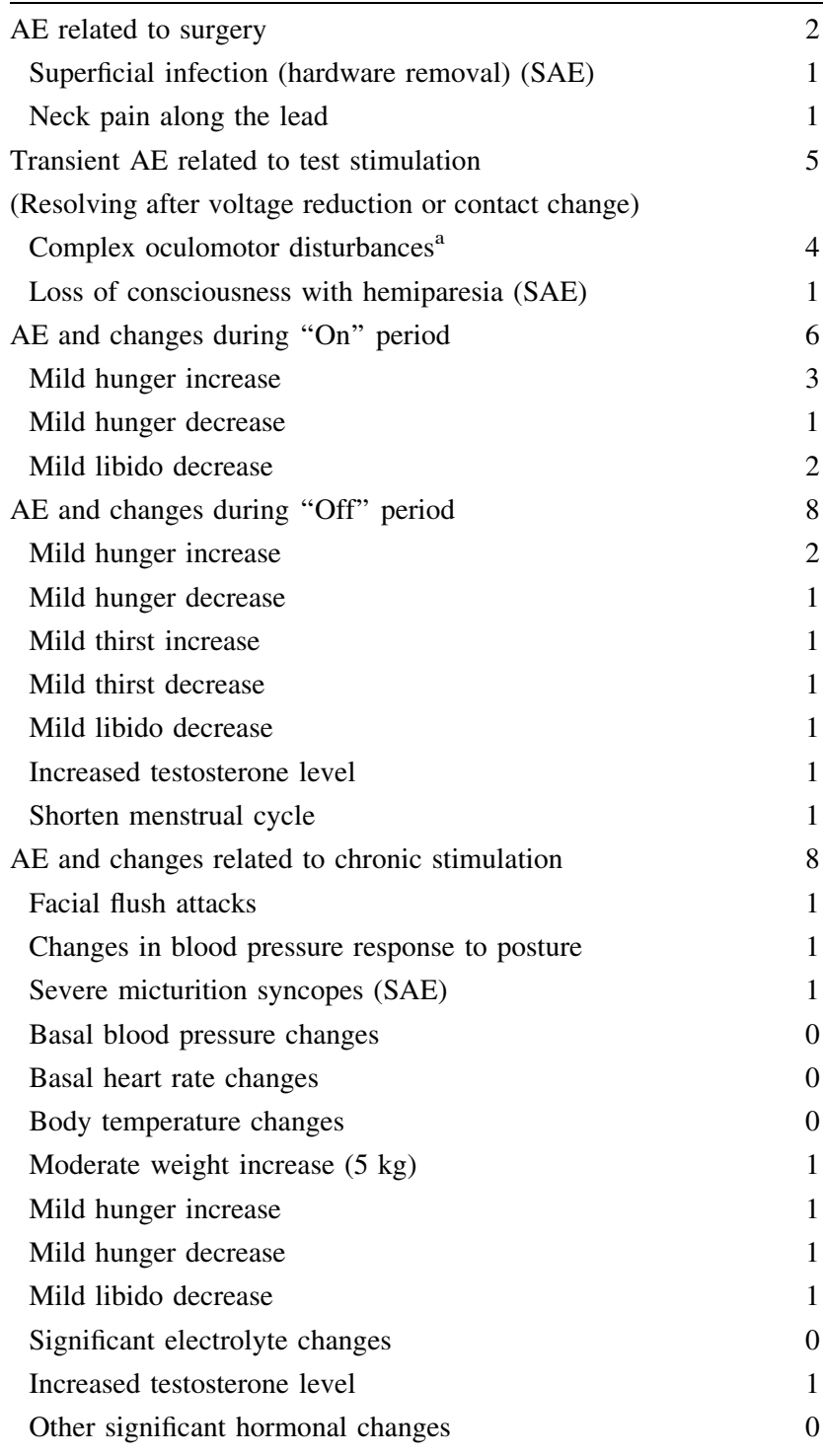

$S A E$ serious adverse event

a Three patients reported a transient diplopia, one reported an impairment of gaze fixation without objective oculomotor paresis

Acknowledgments We thank Sophie Tezenas du Montcel who kindly reviewed the statistical analysis. Study supported by grant from the Programme Hospitalier de Recherche Clinique and promoted by the Centre Hospitalier Universitaire de Nice. Stimulators have been purchased from Medtronic, which had no role in the study, but provided funds for the meetings of the investigators.

\section{References}

1. Headache Classification Committee of the International Headache Society (1988) Classification and diagnosis criteria for headache disorders, cranial neuralgias and facial pain. Cephalalgia 8(Suppl 19):1-96 
2. Headache Classification Committee of the International Headache Society (2004) The international classification of headache disorders. Cephalalgia 24(Suppl 1):1-160

3. Goadsby P, Lipton R (1997) A review of paroxysmal hemicranias, SUNCT syndrome and other short-lasting headaches with autonomic features, including new cases. Brain 120:193-209

4. Goadsby P (2002) Pathophysiology of cluster headache: a trigeminal autonomic cephalalgia. Lancet Neurol 1:251-257

5. May A, Bahra A, Buchel C, Frackowiak R, Goadsby P (1998) Hypothalamic activation in cluster headache attacks. Lancet Neurol 352:275-278

6. May A, Ashburner J, Buchel C, McGonigle D, Friston K, Frackowiak R, Goadsby P (1999) Correlation between structural and functional changes in brain in an idiopathic headache syndrome. Nat Med 5:836-838

7. Leone M, Franzini A, Bussone G (2001) Stereotactic stimulation of posterior hypothalamic grey matter for intractable cluster headache. N Engl J Med 345:1428-1429

8. Bartsch T, Pinsker M, Rasche D, Kinfe T, Hertel F, Diener H, Tronnier T, Mehdorn H, Volkmann J, Deuschl G, Krauss J (2008) Hypothalamic deep brain stimulation for cluster headache: experience from a new multicase series. Cephalalgia 28:285-295

9. Franzini A, Ferroli P, Leone M, Broggi G (2003) Stimulation of the posterior hypothalamus for the treatment of chronic intractable cluster headache. Neurosurgery 52:1095-1099

10. Leone M, Proietti Cecchini A, Franzini A, Broggi G, Cortelli P, Montagna P, May A, Juergens T, Cordella R, Carella F, Bussone G (2008) Lessons from 8 years' experience of hypothalamic stimulation in cluster headache. Cephalalgia 28:789-797

11. Schoenen J, Di Clemente L, Vandenheede M, Fumal A, De Pasqua V, Mouchamps M, Remacle J-M, Maertens de Noordhout A (2005) Hypothalamic stimulation in chronic cluster headache: a pilot study of efficacy and mode of action. Brain 128:940-947

12. Starr P, Barbaro N, Raskin N, Ostrem J (2007) Chronic stimulation of the posterior hypothalamic region for cluster headache: technique and 1-year results in four patients. J Neurosurg 106:999-1005

13. Owen S, Green A, Davies P, Stein J, Aziz T, Behrens T, Voets N, Johansen-Berg H (2007) Connectivity of an effective hypothalamic surgical target for cluster headache. J Clin Neurosci 14:955-960

14. Bartsch T, Paemeleire K, Goadsby P (2009) Neurostimulation to primary headache disorders. Curr Opin Neurol 22:262-268
15. Lanteri-Minet M, Autret A, Baudesson G, Bousser M, Creach' C, Donnet A, Dousset V, Fabre N, Giraud G, Guégan-Massardier E, Guy N, Lucas C, Massiou H, Mick G, Navez M, Pradalier A, Radat F, Valade D, Geraud G, on behalf of the Société Francaise d'Etudes des Migraines et Céphalées (2005) French survey network on headaches and facial pains. In: Olesen J (ed) Classification and diagnosis of headache disorders. Oxford University Press, New York, pp 287-293

16. Lipton R, Micieli G, Russell D, Solomon S, Tfelt-Hansen P, Waldenlind E (1995) Guidelines for controlled trials of drugs in cluster headache. Cephalalgia 15:452-462

17. Jones B, Kenward M (2003) Design and analysis of cross-over trials. Chapman \& Hall, London

18. Diener H, Schorn C, Bingel U, Dodick D (2008) The importance of placebo in headache research. Cephalalgia 28:1003-1011

19. Leone M, Franzini A, Broggi G, Bussone G (2006) Hypothalamic stimulation for intractable cluster headache: long-term experience. Neurology 67:150-152

20. Cortelli P, Guaraldi P, Leone M, Pierangeli G, Barletta G, Grimaldi D, Cevoli S, Bussone G, Baruzzi A, Montagna P (2007) Effect of deep brain stimulation of the posterior hypothalamic area on the cardiovascular system in chronic cluster headache patients. Eur J Neurol 14:1008-1015

21. Black D, Bordini C, Russel D (2006) Symptomatology of cluster headaches. In: Olesen J, Goadsby P, Ramadan N, Tfelt-Hansen P, Welch K (eds) The headaches, 3rd edn. Lippincott Williams and Wilkins, Philadelphia, pp 789-796

22. Goadsby P, Schoenen J, Ferrari M, Silberstein S, Dodick D (2006) Towards a definition of intractable headache for use in clinical practice and trials. Cephalalgia 26:1168-1170

23. Leone M, May A, Franzini A, Broggi G, Dodick D, Rapoport A, Goadsby P, Schoenen J, Bonavita V, Bussone G (2004) Deep brain stimulation for intractable chronic cluster headache: proposals for patient selection. Cephalalgia 24:934-937

24. Starr P (2008) Commentary on Leone et al., lessons from 8 years' experience of hypothalamic stimulation in cluster headache. Cephalalgia 28:798

25. Magis D, Allena M, Bolla M, De Pasqua V, Remacle J, Schoenen J (2007) Occipital nerve stimulation for drug-resistant chronic cluster headache: a prospective pilot study. Lancet Neurol 6:314321

26. Burns B, Watkins L, Goadsby P (2007) Treatment of medically intractable cluster headache by occipital nerve stimulation: longterm follow-up of eight patients. Lancet 369:1099-1106 